Historic, Archive Document

Do not assume content reflects current scientific knowledge, policies, or practices. 

(US)

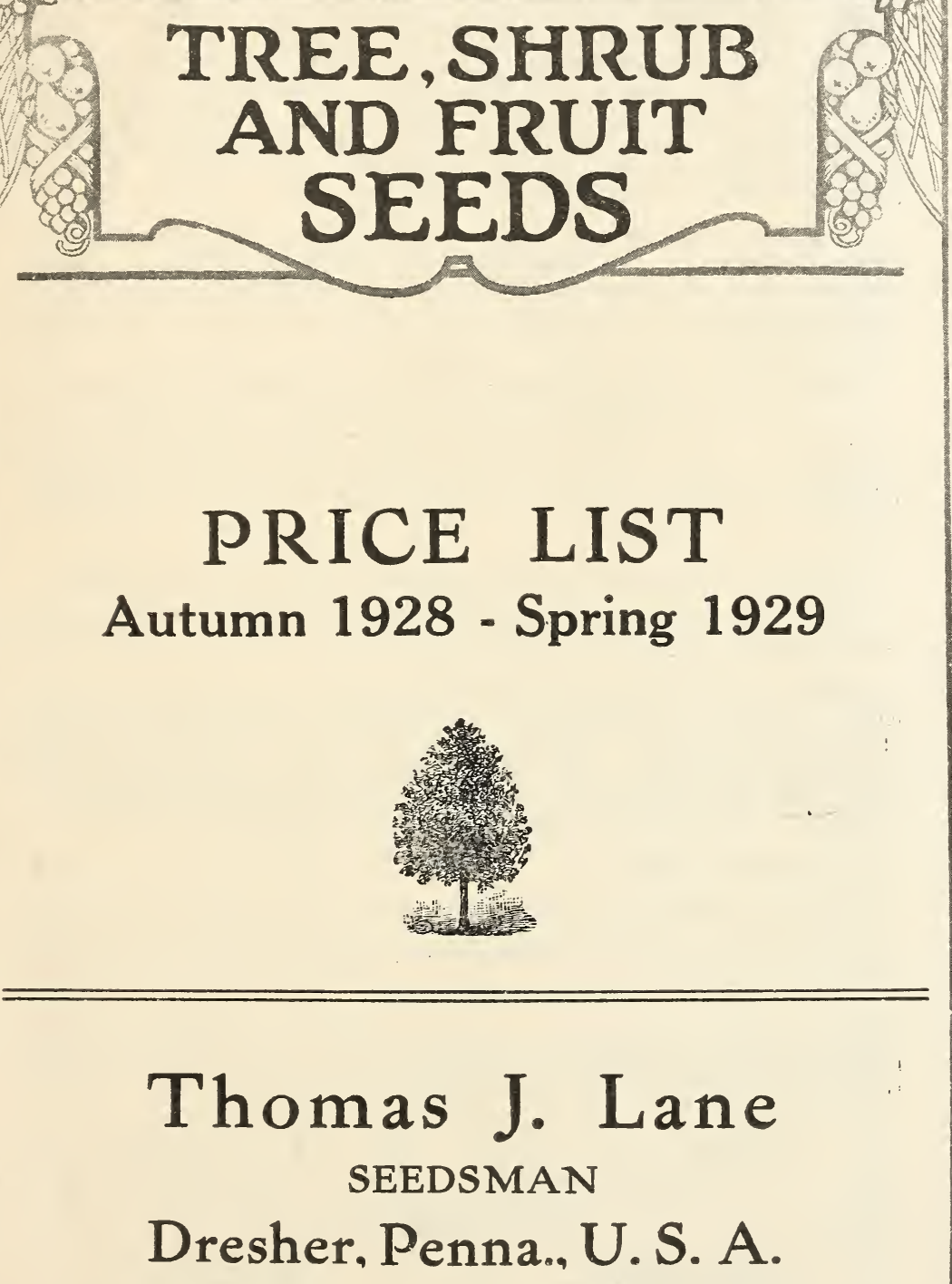




\section{Conditions and Terms}

All offers and acceptances of orders are subject to my receiving the full quantity for which I have placed orders with my growers and collectors. Failing to get these amounts, I reserve the right to reduce proportionately all orders that have been booked; or fill orders in rotation received while supply lasts.

The prices in this list are based on an average crop and normal cost of collecting and preparing the seed. If though unforseen contingencies the cost of any item is extraordinarily increased I reserve the privilege of proportionately advancing the price of such items of accepted orders, subject to the buyer's option to pay the increased price or cancel those parts of his order.

I give no warranty, express or implied, as to description, quality, productiveness, or any other matter of any seeds, bulbs or plants I send out, and will not be in any way responsible for the crop. If the purchaser does not accept the goods on these terms they are at once to be returned.

\section{All Claims Must be Made Upon Receipt of Goods}

AFTER GOODS ARE PLACED ON BOARD CARS HERE, I AS. SUME NO RESPONSIBILITY FOR THEIR SAFE DELIVERY OR ANY INJURY CAUSED BY DELAY IN DELIVERY BY THE TRANSPORTATION COMPANY.

\section{SACKS AND BOX AND PACKING CHARGED ADDITIONALLY.}

Not less than one-half pound will be supplied at pound rates nor one-quarter bushel at bushel rates.

When seeds are to go by mail, postage is charged at Parcel Post rates in U.S. A. Consult your postmaster as to your zone and allow charges accordingly. Foreign countries 12c. per pound for postage.

SATISFACTORY ARRANGEMENTS FOR PAYMENT MUST ACCOMPANY ORDERS FROM UNKNOWN CORRESPONDENTS.

All P. O. orders payable at Dresher, Pa., U. $\Im$. A.

Terms-Net Cash.

SPECIAL PRICES WILL BE QUOTED ON LARGE QUANTITIES. Cable Address "LANE AMBLER" A. B. C. Code 4th and 5th Edition.

All orders will receive my best attention. Packing for shipmen: will receive special care and seeds will be packed in a manner that will permit their reaching destination in perfect order.

\section{Thomas J. Lane SEEDSMAN Dresher, Penna.}




\section{General Information and Suggestions For Planting}

Seeds to germinate must have the proper conditions of heat and moisture. These conditions vary greatly with different seeds. Experience and close attention are necessary to successful propagation and the propagator must be a close observer of the wants and habits of trees and plants and be able to gather knowledge from what he may see. Owing to certain conditions of moisture, temperature and time which they do not always meet with after sowing, seeds sometimes fail to appear the first season. Examine them at close of Spring, and if found sound do not disturb tho-n, and their germination may be looked for the season following.

Seeds of deciduous trees and shrubs are best sown in the Fall, pines and similar evergreens in Spring, but deciduous ones will grow very well sown in Spring if kept in good condition through the Winter. When sown in Spring seeds of a liard, bony nature, such as the yellow locust, are best soaked in hot water for a day or two and then sown at once. Seeds of maples, ash, magnolia, beech, all nut trees, as well as many other kinds require to be kept slightly moist over Winter if not sown in Fall; on the other hand, catalpa and some others may be kept quite dry until Spring comes.

Junipers, hollies and a few similar seeds remain a full year before sprouting. It is just as well, therefore, to keep them in a box of soil until the next Autumn, then sow them.

If the planting is done in the Fall it is better to mulch the ground with straw, leaves, or any like material; this will prevent baking of the soil after the Spring rains, and keep it in a nice mellow condition. The mulching should be removed in the Spring, or, at least so much that it will not interfere with the growth of the young seedlings.

The seed of evergreens is always found in cones, with the exception of the juniper and yew, which pruduce small berries; hence, the general name applied to evergreens of coniferae, or cone-bearing.

The soil most suitable for raising conifers is a light sandy loam, rich in vegetable matter, but entirely free from rank manures. The soil should be deeply spaded and thoroughly pulverized. Then lay off in beds four feet wide. and any desirable length, with paths of one foot between. The beds should be higher in the middle, the sides being but little higher than the paths. Tle seed is usually sown broadcast and then carefully and lightly raked in; but some prefer sowing in irills a few inches apart, then rolling, or pressing with back of the sparie, and sifting over them a very light covering of oneeighth to one-quarter of an inch of soil. The ground should be prepared the Fall before, and the seed put in at the earliest possible moment in the Spring. Most failures in growing evergreen seedlings arise from not having the seed beds partially shaded. If hot sunshine falls on them while the seeds are swelling. and cold follows, a large proportion will rot before they apnear above the ground. When seedlings are raised extensively artificial arbors are made high enough to work under. When the growing is done on a less exten. sive scale, stakes are driven at convenient distances along the sides of the beds to which boards six inches wide are nailed, to support screens for shading the young seedlings. The lower enge of these boards should be hut four or five inches above the surface of the ground. to allow the air to circulate freely over the beds. Lath screens are very satisfactory. 
The following suggestions may be of service to the nurseryman, but should be changed as conditions may require.

\begin{tabular}{|c|c|c|c|}
\hline $\begin{array}{l}\text { Andromeda } \\
\text { Azalea }\end{array}$ & Kalmia & Rhododendron & $\begin{array}{l}\text { Sow in peat moss } \\
\text { or fine leaf mould } \\
\text { in well protected } \\
\text { seed bed. }\end{array}$ \\
\hline $\begin{array}{l}\text { Carpinus } \\
\text { Edgeworthia }\end{array}$ & Prunus & $\begin{array}{l}\text { Silver maple } \\
\text { Quercus } \\
\text { Ulmus }\end{array}$ & $\begin{array}{l}\text { Sow as soon as } \\
\text { possible after } \\
\text { seeds are re- } \\
\text { ceived. }\end{array}$ \\
\hline $\begin{array}{l}\text { Acer } \\
\text { Aesculus } \\
\text { Benzoin } \\
\text { Calycanthus } \\
\text { Caragana } \\
\text { Carpinus } \\
\text { Celtis } \\
\text { Chionanthus }\end{array}$ & $\begin{array}{l}\text { Cinnamommum } \\
\text { (in warmer } \\
\text { weather) } \\
\text { Cornus } \\
\text { Corylus } \\
\text { Fraxinus } \\
\text { Juglans } \\
\text { Liriodendron }\end{array}$ & $\begin{array}{l}\text { Ligustrum } \\
\text { Pinus Strobus } \\
\text { Prunus } \\
\text { Roses } \\
\text { Taxodium } \\
\text { Tilia } \\
\text { Rhus } \\
\text { Syringa }\end{array}$ & $\begin{array}{l}\text { Sow in Autumn } \\
\text { or when seed ar- } \\
\text { rives. }\end{array}$ \\
\hline $\begin{array}{l}\text { Actinidia } \\
\text { Akebia } \\
\text { Berberis } \\
\text { Catalpa } \\
\text { Cercidiphyllum } \\
\text { Clematis }\end{array}$ & $\begin{array}{l}\text { Desmodium } \\
\text { Diospyrus } \\
\text { Fagus } \\
\text { Gleditschia } \\
\text { Juniperus } \\
\text { Kerria }\end{array}$ & $\begin{array}{l}\text { Paeonia } \\
\text { Pines } \\
\text { Pyrus } \\
\text { Sambucus } \\
\text { Sophora } \\
\text { Staphylea }\end{array}$ & $\begin{array}{l}\text { Sow in e arly } \\
\text { spring. }\end{array}$ \\
\hline $\begin{array}{l}\text { Abies } \\
\text { Ampelopsis } \\
\text { Aralia } \\
\text { Aucuba } \\
\text { Betula } \\
\text { Biota } \\
\text { Buxus } \\
\text { Celastrus } \\
\text { Cercis } \\
\text { Chamaecyparis } \\
\text { Clethra } \\
\text { Cryptomeria } \\
\text { Cydonia }\end{array}$ & $\begin{array}{l}\text { Euonymus } \\
\text { Ginkgo } \\
\text { Hibiscus } \\
\text { Ilex } \\
\text { Larix } \\
\text { Lindera } \\
\text { Liquidambar } \\
\text { Magnolia } \\
\text { Morus } \\
\text { Nandina } \\
\text { Paulowina } \\
\text { Photina } \\
\text { Picea }\end{array}$ & $\begin{array}{l}\text { Pinus } \\
\text { Pueraria } \\
\text { Rhodotyphus } \\
\text { Robinia } \\
\text { Sciadopitys } \\
\text { Spirea } \\
\text { Styrax } \\
\text { Taxus } \\
\text { Thujopsis } \\
\text { Tsuga } \\
\text { Viburnum } \\
\text { Wisteria }\end{array}$ & $\begin{array}{l}\text { Sow in spring af- } \\
\text { ter d a } \mathrm{ng} r \text { of } \\
\text { frost is past and } \\
\text { soil is warm. }\end{array}$ \\
\hline $\begin{array}{l}\text { Carya } \\
\text { Crataegus } \\
\text { Juniperus }\end{array}$ & $\begin{array}{l}\text { Magnolia } \\
\text { Rhodotyphus } \\
\text { Taxus }\end{array}$ & $\begin{array}{l}\text { Tilia } \\
\text { Viburnum }\end{array}$ & $\begin{array}{l}\text { Stratify b e f o r e } \\
\text { sowing; s e ed s } \\
\text { germinate slowly. }\end{array}$ \\
\hline $\begin{array}{l}\text { Buxus } \\
\text { Camellia } \\
\text { Cercis } \\
\text { Gleditschia }\end{array}$ & $\begin{array}{l}\text { Ilex } \\
\text { Photinia } \\
\text { Pueraria } \\
\text { Robinia }\end{array}$ & $\begin{array}{l}\text { Rosa } \\
\text { Sophora }\end{array}$ & $\begin{array}{l}\text { Soak in hot water } \\
\text { just before plant- } \\
\text { ing. }\end{array}$ \\
\hline $\begin{array}{l}\text { Ampelopsis } \\
\text { Aucuba } \\
\text { Berberi: } \\
\text { Cinnamominm } \\
\text { Euonymus }\end{array}$ & $\begin{array}{l}\text { Ginkgu } \\
\text { Hedera } \\
\text { Ligustrum } \\
\text { Lindera } \\
\text { Magnolia }\end{array}$ & $\begin{array}{l}\text { Prunus } \\
\text { Rosa } \\
\text { Sapindus } \\
\text { Viburnum }\end{array}$ & $\begin{array}{l}\text { Wash off thick } \\
\text { pulp before strati. } \\
\text { fying or sowing. }\end{array}$ \\
\hline
\end{tabular}

Practical Plant Propagation by Hottes, is a very helpful book for planters. It contains much valuable information. Price $\$ 2.15$ Postpaid. 


\section{Evergreens}

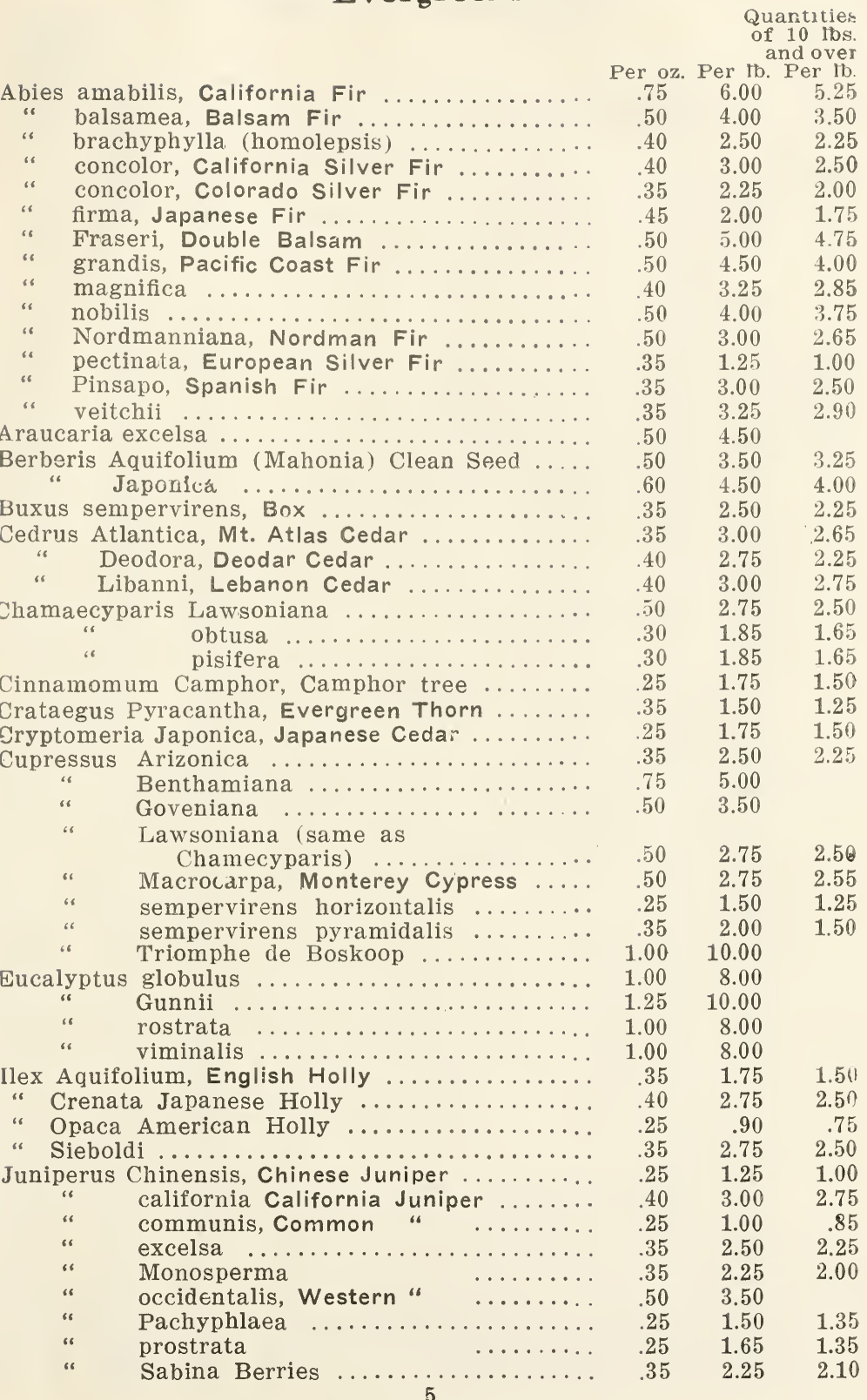




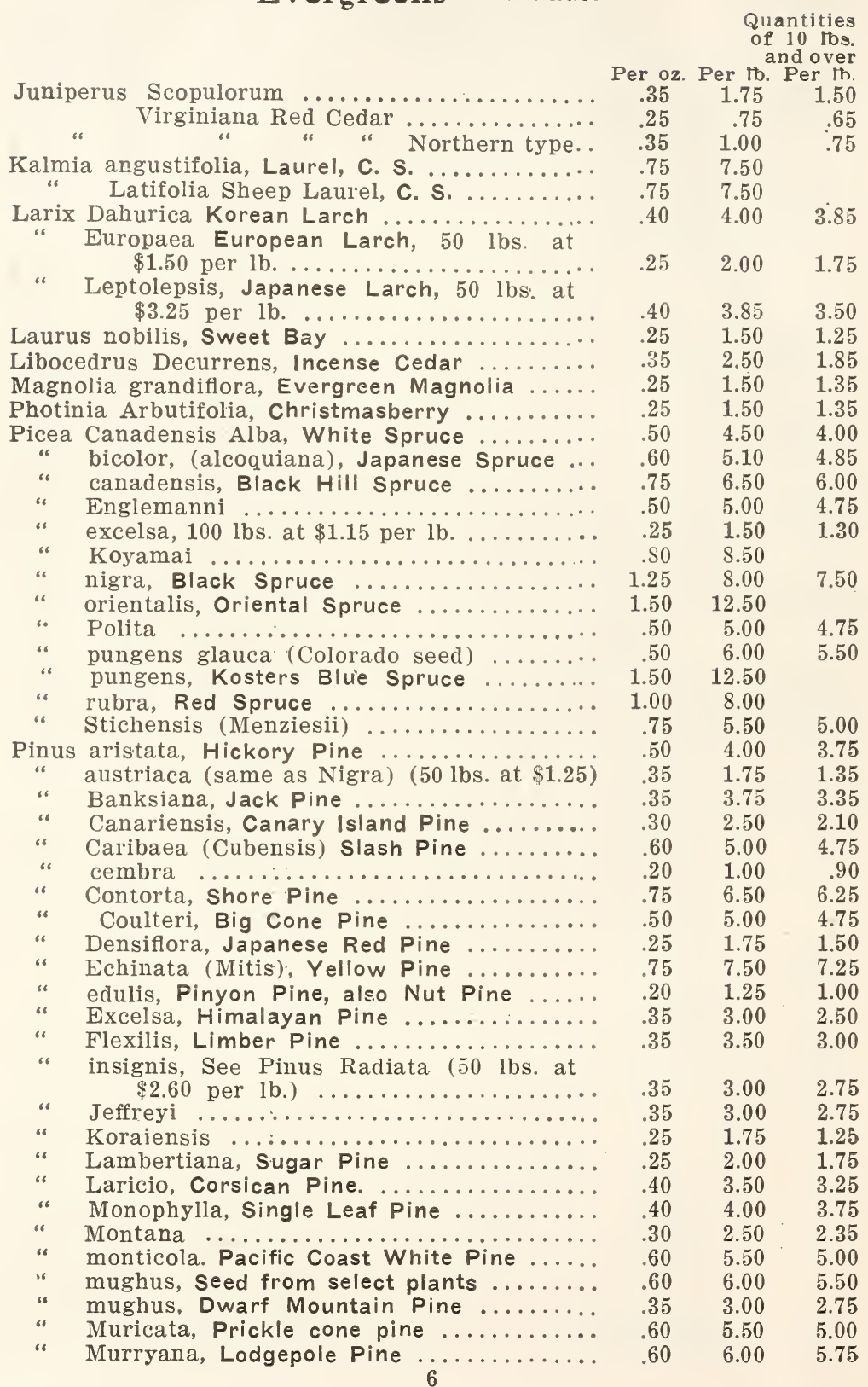




\section{Evergreens-Continued}

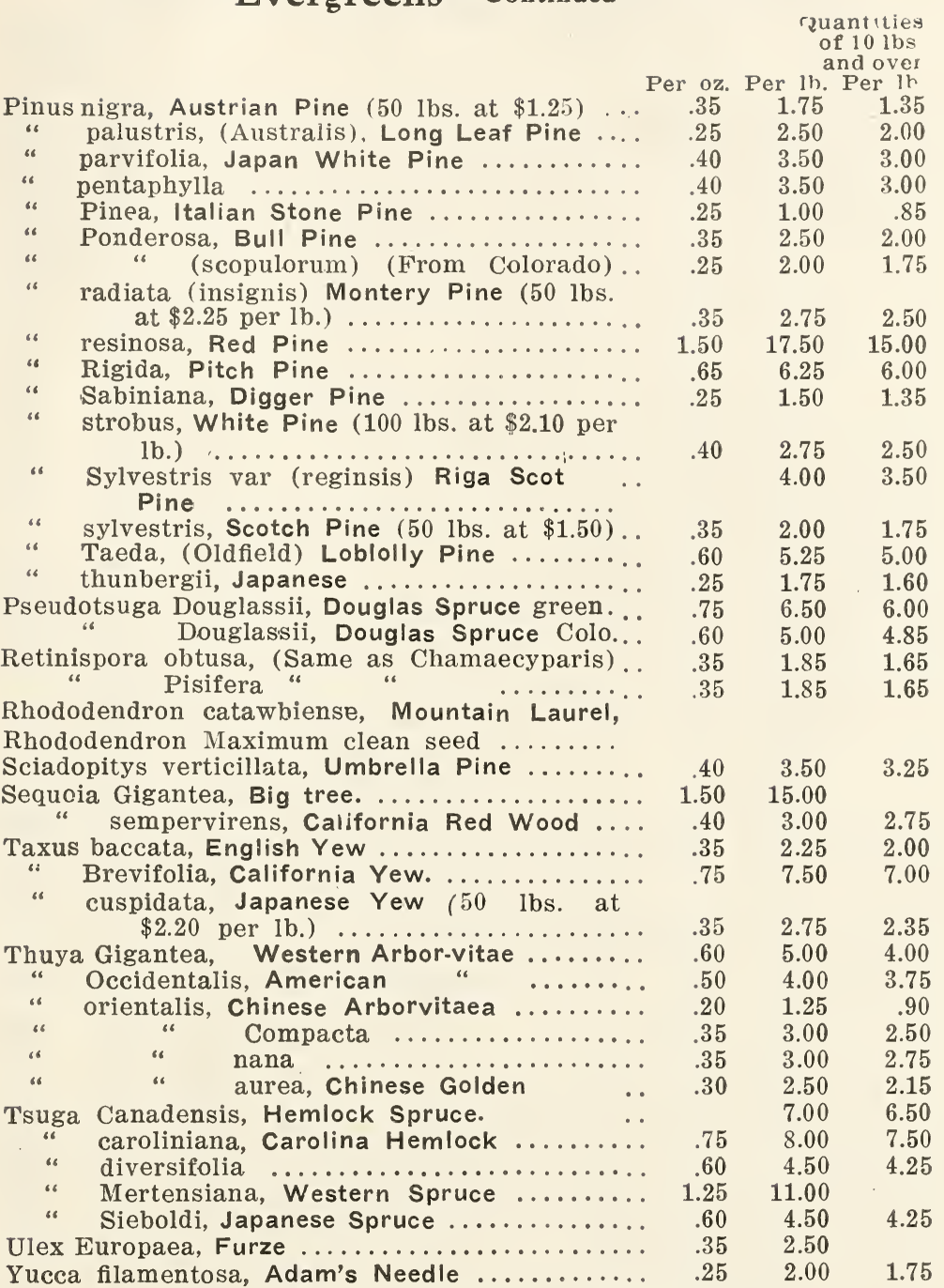

\section{Deciduous Trees and Shrubs}

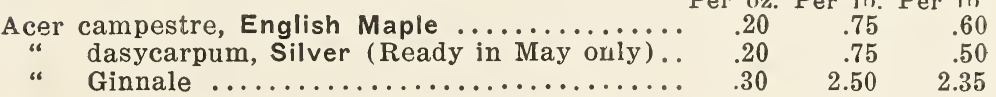




\section{Deciduous Trees and Shrubs--Continued}

Quantities

of $10 \mathrm{lbs}$.

and over

Per oz. Per lb. Per lb.

Acer Japonicum ...................................... $2.75 \quad 2.35$

Negundo, Ash Leaf Maple ............. .25 $\quad 1.25 \quad 1.00$

macrophyllum, Oregon Maple .......... $\quad .20 \quad .60 \quad .40$

palmatum ( 50 lbs. at $\$ 2.10$ per lb.) .... $\quad .35 \quad 3.00 \quad 2.50$

Palmatum, Atropurpureum, Japan Blood.. $\begin{array}{llll}1.25 & 12.00 & 11.50\end{array}$

Platanoides, Norway ................... .25 $\quad .85 \quad .60$

Platanoides, Schwedleri Blood leaf ..... $\quad .25 \quad 2.00 \quad 1.75$

Pennsylvanicum (Striatum) Striped leaf.. $\quad .25 \quad 2.00 \quad 1.85$

rubrum, Red Maple (Ready in May only).. $\quad \begin{array}{lll}50 & 3.00 & 2.85\end{array}$

saccharum. Sugar Maple .............. $25 \quad 1.00 \quad .85$

spicatum, Mountain " $\ldots \ldots \ldots \ldots \ldots \ldots . .30 \quad 2.75 \quad 2.50$

striatum, (same as Pennsylvanicum) $\ldots . . .250 .25 \quad 2.00 \quad 1.85$

Aesculus hippocastanum, Horse Chestnut .... $\quad .20 \quad .60 \quad .40$

" rubicunda, Red Flowering ........ .25

Ailanthus glandulosa, Tree of Heaven ........ .2.

Amorpha Fruticosa Indigo Shrub ...............

Andromeda (Pieris) Japonicum, Clean Seed .... 1.50

Aralia Spinosa, Angelica tree............. .35

Aronia Arbutifolia, Red Chokeberry ........ $\quad .20$

Melanocarpa, Black Chokeberry .......... .15

Asimina triloba, Pawpaw ............... .25

A ucuba Japonica .................. $\quad .30$

Azalea Calendulacea, Clean Seed $\quad$. 1.00

hinodigeri, " "

Mollis, " "

Vudiflora " "

Tiscosa " "

$1.50 \quad 1.25$

$1.00 \quad .75$

$.65 \quad .50$

12.00

$3.00 \quad 2.50$

$1.25 \quad 1.10$

$1.00 \quad .85$

$1.35 \quad 1.10$

$2.00 \quad 1.85$

8.00

10.00

8.00

8.00

Benzoin Aestivale, Spicebush. ................. $\quad .25$

Berberis Thunbergii, Japanese Barberry berries. $\quad .25 \quad 1.50$

1.10

$1.50 \quad 1.25$

$3.50 \quad 3.10$

$\begin{array}{llllr}\text { vulgaris, Common Barberry } \ldots \ldots \ldots \ldots \ldots & .25 & 1.10 & .90 \\ \text { " Purpurpea, Purple Barberry . } & .25 & 2.00 & 1.75\end{array}$

$.75 \quad .65$

$2.50 \quad 2.10$

$2.25 \quad 1.85$

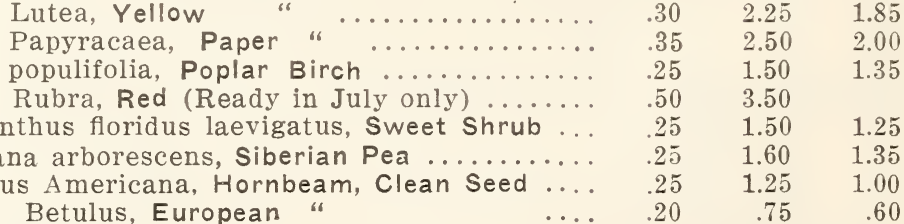

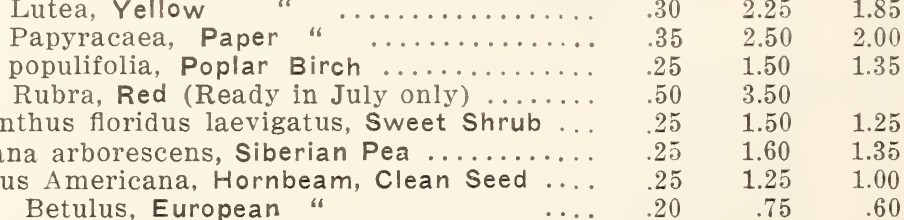

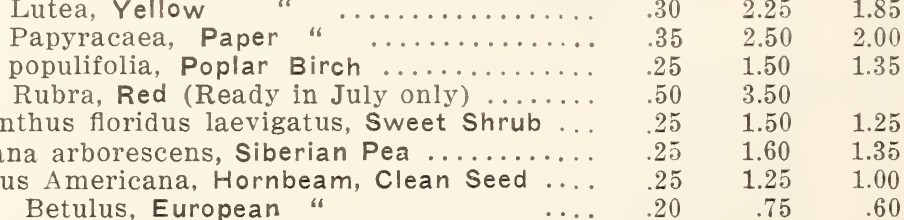

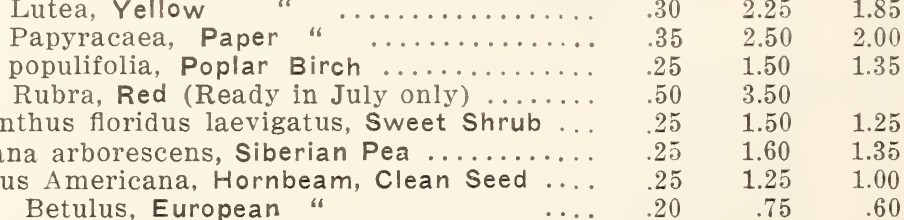

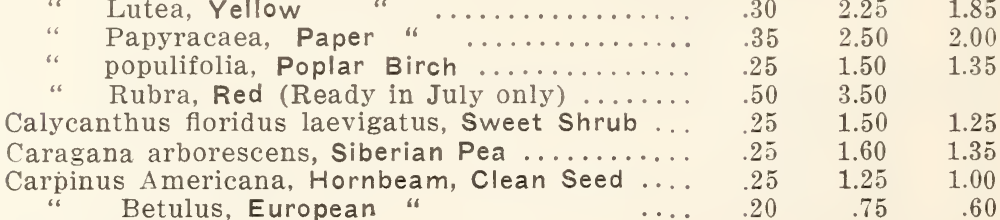

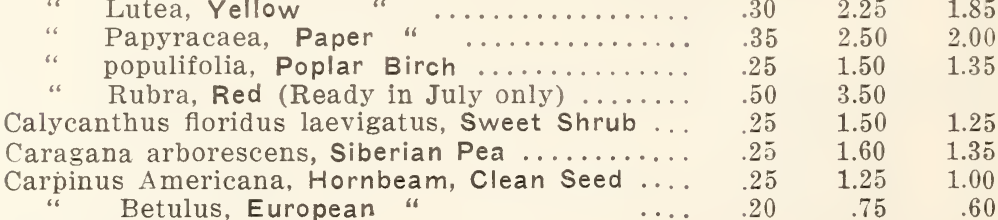

Carya, (same as Hicoria)

Castanea Americana, American Sweet

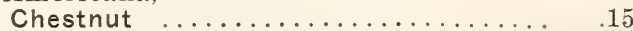

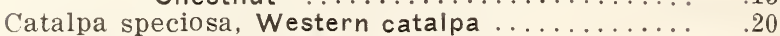

Ceanothus Americanus, Clean Seed ......... $\quad .50$

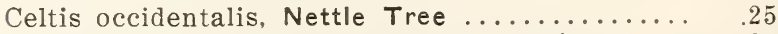

Cephalanthus Occidentalis, Button Bush ...... $\quad .20$

Cercidyphyllum Japonicum, Katsura tree ..... .75

Cercis Canadensis, Judas Tree .............. .25 


\section{Deciduous Trees and Shrubs-Continued}

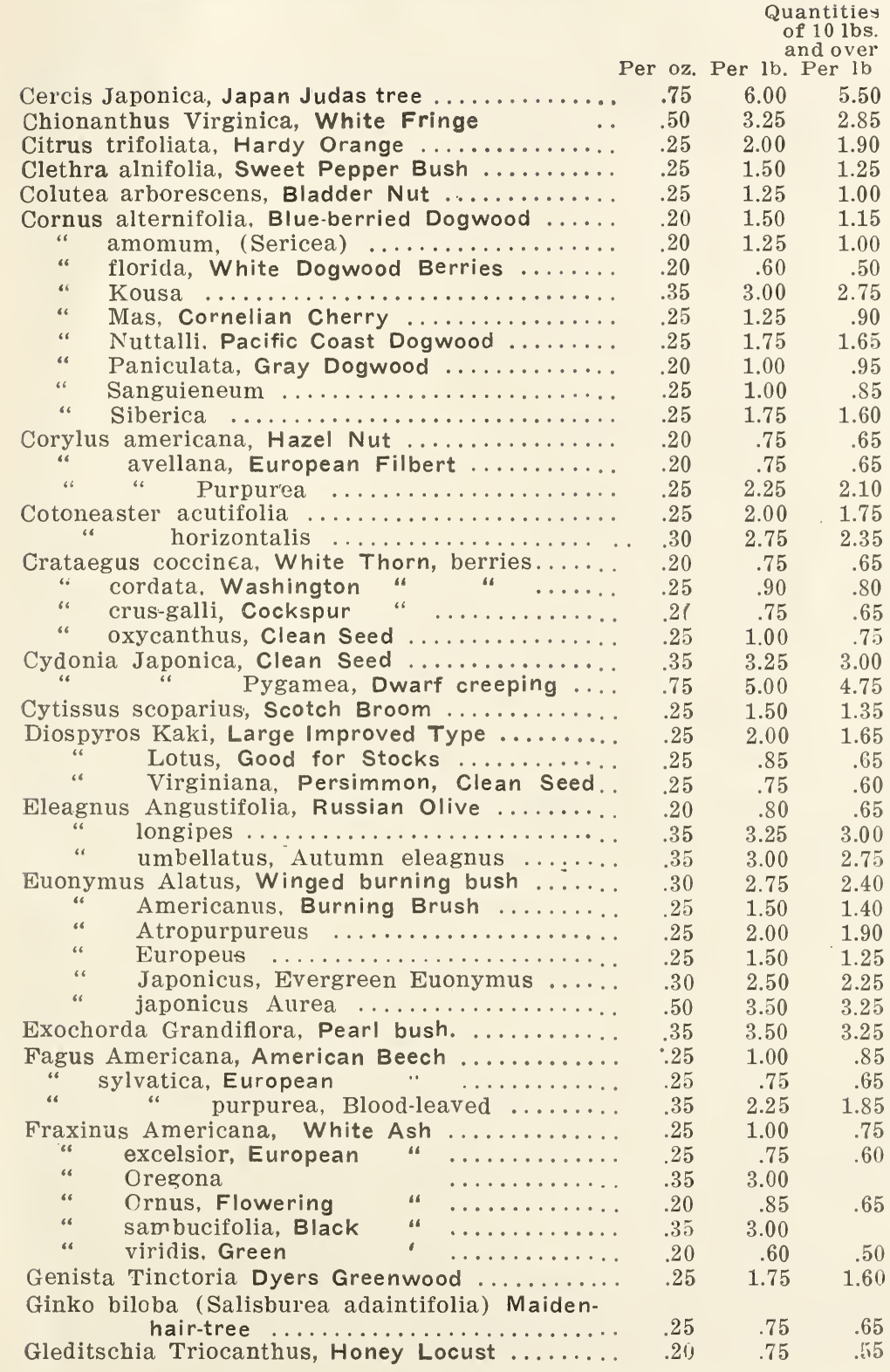




\section{TNOMAS T: LANE DRESHER Pa. \\ Deciduous Trees and Shrubs-Continued} Quantities of $10 \mathrm{lbs}$ and over

Per oz. Per ib. Per Ib.

Gymocladus Canadensis, Kentucky Coffee .... Halesia tetraptera, snowdrop Tree ........... Hamamelis Virginica, Witch Hazel ........... Hibuscus Syriacus, Althea clean seed ..........

Hicoria Alba (Carya Tomentosa) Mockernut Cordiformis (Carya Amara) Bitternut

Glabra (C. porcina) Pignut ........... .10

Lacinosa (C. Sulcaat) Shellbark ....... .10

.20

.25

.25

.35

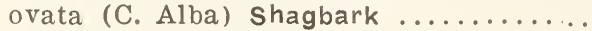

Pecan (C. olivaeformis) Pecan ........

Eiippophaea rhamnoides, Sea Buckthorn..........

llex decidua $\ldots \ldots \ldots \ldots \ldots \ldots \ldots \ldots \ldots \ldots \ldots . \ldots \ldots$

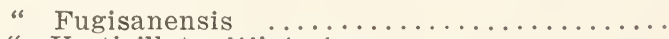

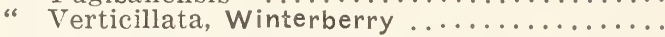

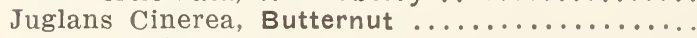

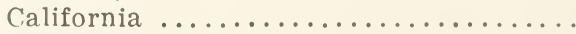

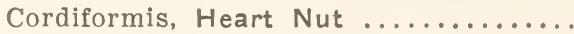

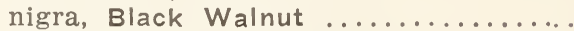

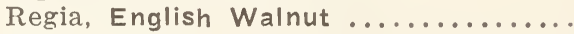

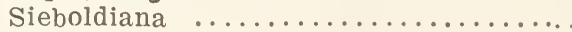

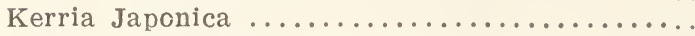

Koelteruria Paniculata, Varnish Tree ..........

Iarix Europea, European Iarch (50 lbs. @

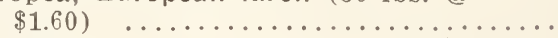

“ leptolepsis (50 lbs. at $\$ 3.10$ per 1b.) .....

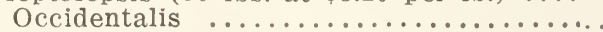

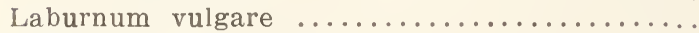

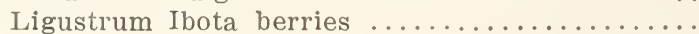

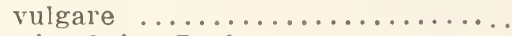

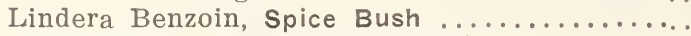

Liquidambar styraciflua, Sweet Gum ..........

Liriodendron tulipifera, Tulip Tree ...........

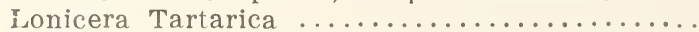

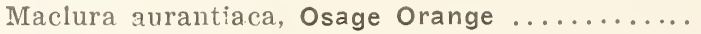

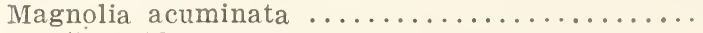

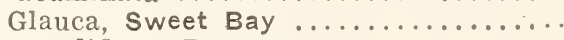

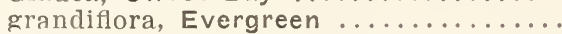

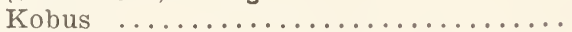

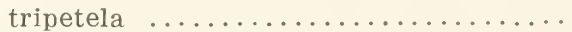

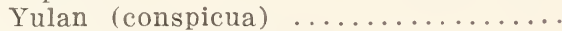

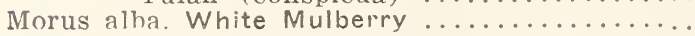

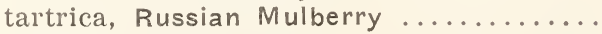

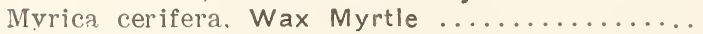

Negundo Aceroides, Ash-leaved Maple ........

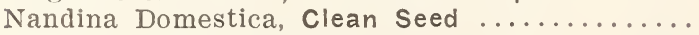

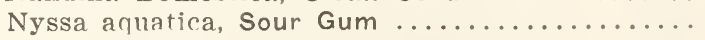

" multiflora (sylvatica) $\ldots \ldots \ldots \ldots \ldots \ldots$

oxydendrum arborcum (Andromeda) clean seed

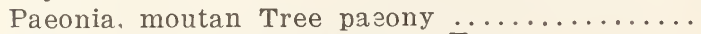

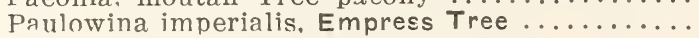

Pieris japonica (Andromeda) Clean seed ......
.75

1.50

2.00

1.25

.65
1.15

1.75

1.10

$.25 \quad .16$

$.25 \quad .18$

$.25 \quad .17$

$.60 \quad .50$

$1.50 \quad 1.25$

$1.50 \quad 1.15$

25.00

1.25

.25

.75

.65

.20

.65

.65

3.75

.75

2.00

4.00

7.50

1.25

.50

1.00

1.25

3.50

1.00

3.50

1.50

2.00

3.50

1.75

2.25

2.00

7.50

2.00

2.50

1.50

.65

2.50

1.25

1.25

1.25

3.75

8.00

1.25

10.00
1.00

.15

.60

.50

.14

.60

.50

2.85

.60

1.75

3.25

7.00

1.10

.40

.65

1.00

3.25

.75

1.25

1.75

3.25

1.60

2.00

1.50

1.85

2.25

1.20

.50

2.25

1.00

1.00

1.00

3.50

7.50

1.00 
Platanus occidentalis, American Plane ......... $\quad .20 \quad 1.00 \quad .75$

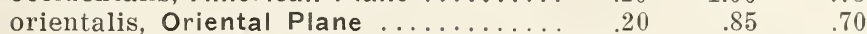

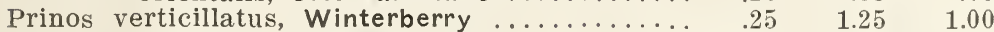

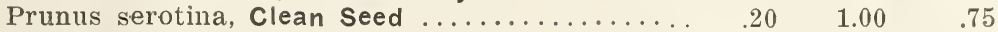

Ptelea trifoliata, Hop Tree ............... .25 $\quad 1.00 \quad .90$

Pyrus americana (same as sorbus) ........... $.25 \quad 1.35 \quad 1.15$

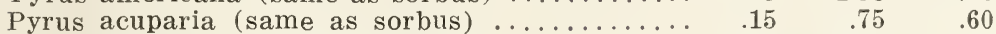

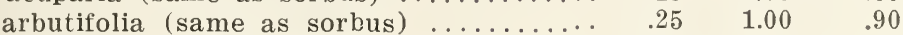

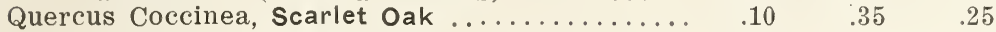

Falcata, Spanish Oak ..............................

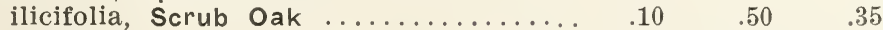

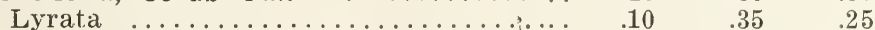

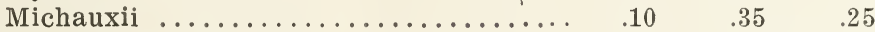

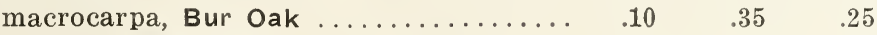

Nigra, Black Jack ....................... $10 \quad .40 \quad .30$

palustris, Pin Oak (50 lbs. @ .30) ..... $\quad .10 \quad .40 \quad .35$

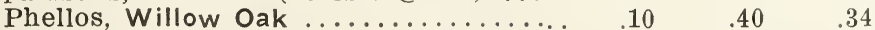

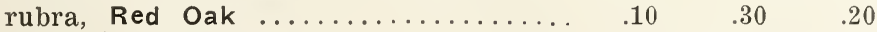

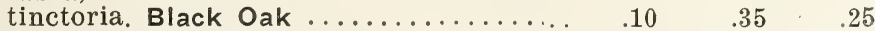

Rhamnus Cathartica, Buckthorn ........... $20 \quad 1.25 \quad 1.00$

$1.75 \quad 1.60$

$1.50 \quad 1.25$

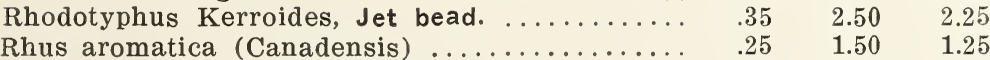

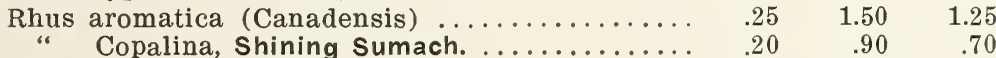

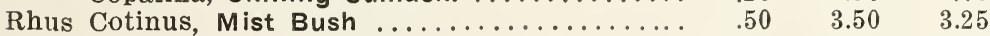

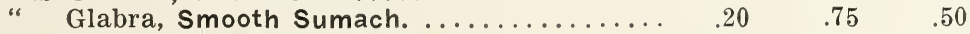

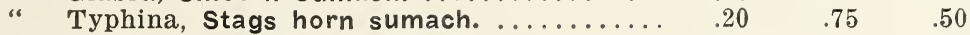

Ribes sanguieneum, Flowering Currant ........ $\quad .50 \quad 4.00 \quad 3.50$

Robina Pseudacacia, Yellow or Black Locust ... $\quad .20 \quad .85 \quad .60$

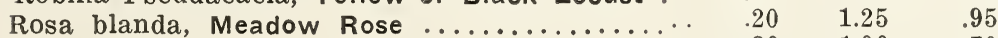

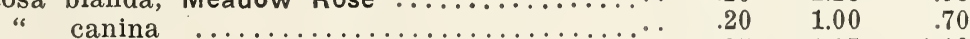

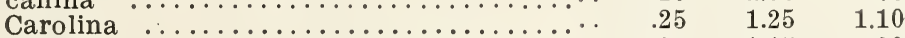

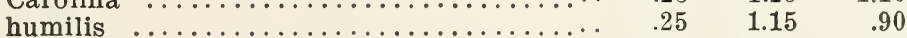

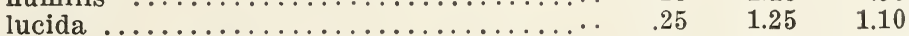

Multiflora Japonica Berries, American.... $\quad .20 \quad 1.25 \quad 1.00$

Rubignosa. Clean Seed, European.. .25 .25 $1.25 \quad 1.00$

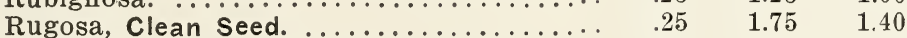

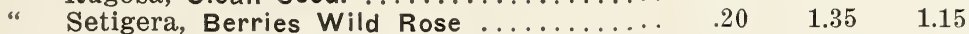

Salisaburia Adiantifolia (same as Gingko) $\ldots \ldots \ldots . \quad .20 \quad .60 \quad .50$

Sambucus Canadensis, Elder, Clean Seed . . . . . . $\quad .50 \quad 4.00 \quad 3.75$

Sassafras officinale ....................... .25 $1.50 \quad 1.25$

Sophora Japonica, Pagoda Tree, Clean Seed .... $\quad .20 \quad 1.00 \quad .85$

Sorbus Americana, American Mt. Ash ......... $25 \quad 1.35 \quad 1.15$

Aucuparia, European Mt. Ash ....... $\quad .15 \quad .75 \quad .60$

Spiraea Opulifolia. Clean Seed ............. $40 \quad 4.00$

Syringa dilata, Korean lilac clean seed ...... $1.50 \quad 12.00$ japonica, Japanese Tree Lilac ...... $\quad .75 \quad 8.00 \quad 7.50$ vulgaris, Common Lilac, Cleall Seed.. $\quad .40 \quad 3.50 \quad 3.00$

$.60 \quad 5.00 \quad 4.50$

Taxodium distichum, Deciduous Cypress ......... 


\section{Deciduous Trees and Shrubs-Continued}

\begin{tabular}{|c|c|c|}
\hline \multirow{3}{*}{$\begin{array}{c}\text { Per oz. } \\
.25 \\
20\end{array}$} & \multicolumn{2}{|c|}{$\begin{array}{l}\text { Quantities } \\
\text { of } 10 \text { lbs. } \\
\text { and over }\end{array}$} \\
\hline & $\begin{array}{c}\text { Per lb. } \\
1.50\end{array}$ & $\begin{array}{r}\text { Per lb. } \\
1.10\end{array}$ \\
\hline & 1.00 & .9 \\
\hline .20 & 1.00 & \\
\hline .20 & 1.15 & \\
\hline .25 & 1.25 & \\
\hline .20 & .75 & \\
\hline .75 & 6.00 & 5. \\
\hline .20 & 1.25 & 1. \\
\hline .20 & 1.15 &. \\
\hline .25 & 1.25 & 1. \\
\hline .25 & 1.25 & 1. \\
\hline .25 & 1.25 & \\
\hline .20 & 1.00 & \\
\hline
\end{tabular}

\section{Vines and Climbers}

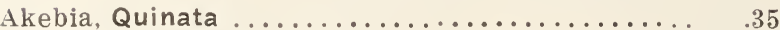

Ampelopsis veitchii, Japan creeper berries .....

" Virginica, Virginia "

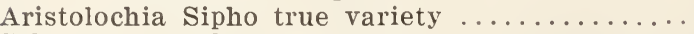

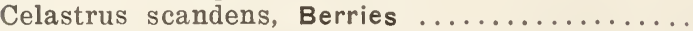

Clematis flammula, sweet Clematis ............

$.25 \quad 1.50 \quad 1.25$
paniculata, real clean seed (this is clean seed, practically free from all foreign matter and not to be confused with inferior quality offered at a less price) .. $\quad .35$

Hedera Helix, Ivy .................... .25

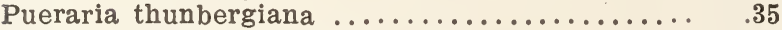

2.25

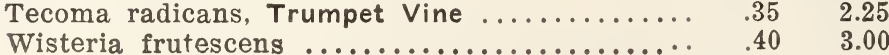

\section{Fruits}

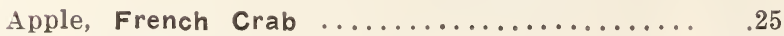

Cherry, Mahaleb clean stones (50 lbs. at .85) ... $\quad .25$

Myrobolan plum (50 lbs. at .60 per lb) .........

Pear, French Pear .................. .35 